\title{
The mechanical behavior of an expansive soil due to long-term seasonal rainfalls
}

\author{
Kai $\mathrm{LI}^{1,2, *}$, Liang Kong ${ }^{1}$, Hossein Nowamooz ${ }^{2}$ and Cyrille Chazallon ${ }^{2}$ \\ ${ }^{1}$ School of Sciences, Qingdao University of Technology, 266033, 11 Fushun Road, China \\ 2 ICUBE, University of Strasbourg, 67084, 24 Boulevard de la Victoire, France
}

\begin{abstract}
Expansive soils, susceptible to be affected by the environmental conditions, expand when water is added and shrink when they dry out. This continuous change in soil volume is able to cause structures built on them to move unevenly and crack. To investigate the hydro-mechanical behavior of unsaturated expansive soils, many laboratory tests on these materials have been carried on and numerous models have also been proposed with a relatively large number of parameters. In this study, a simplified model based on Zarka method has been developed for unsaturated expansive soils. The direct determination of the steady solutions in Zarka analysis is able to replace classic step-by-step method and needs less model parameters. In this context, this paper presents a Zarka-based model to predict the volume change in unsaturated expansive soils under seasonal drought and rainfall cycles and the proposed model is implemented in the finite element code to simulate long-term behavior of a 2D structure consisting of expansive soils and subjected to successive drought and rainfall cycles. Finally, the numerical calculation defines the plastic strain field and the inelastic displacement field of the studied structure.
\end{abstract}

\section{Introduction}

Expansive soil generally contains smectite clayey particles that join other soil components to form aggregates. This material is extensively distributed throughout many regions in the world and it presents a series of threats to lightly loaded structures because it undergoes differential deformations mainly due to variations in moisture content. The climate condition may have a more significant effect on the soil moisture change, particularly evapotranspiration in the drought season and infiltration in the rainfall season. In this context, it is necessary to investigate the hydromechanical behavior of expansive soils to better control their use.

Much research has been devoted to investigating expansive soil modeling. However, the objectives and advantages of these models are somewhat varied. Alonso et al. [1,2] have developed the most accepted model (BExM), able to describe the swelling-shrinkage behavior during wetting-drying cycles based on a formalism for double structures. With the framework of BExM, independent coupled hydro-mechanical mechanism has been considered for each structural level, including separate measures of micro- and macromechanical effective stresses [3]. A number of recent models have extended BExM formulation to the complex stress paths, and the coupling effects between thermal, hydraulic and mechanical behavior have also been taken into account by several models [3-8]. However, a relatively large number of parameters, difficult to quantify, are introduced to the coupling functions between micro- and macro-structural levels. Therefore, alternative and simplified models are necessary.

This study focuses on the long-term behavior of expansive soils due to cyclic rainfall infiltration and evapotranspiration (wetting and drying). To simulate this behavior, the following research aspects are included in the research: 1) Development of a simplified model based on the Zarka method for unsaturated expansive soils subjected to suction cycles; 2) Implementation of the proposed model in a finite element code CAST3M to simulate the settlement of a shallow foundation constructed on the studied expansive soil.

\section{Modeling of expansive soils}

In this section, a structure of unsaturated expansive soils with an elasto-plastic behavior is considered. Its boundary $\Gamma$ is subjected to the imposed surface forces $F_{i}(x)$ on $\Gamma_{F i}$ partition and the prescribed surface displacements $U_{j}(x)$ on $\Gamma_{U j}$ partition. The body force $X_{j}(x)$ and the initial strain $\varepsilon_{i j}(x, t=0)$ are defined in the volume $V$. Moreover, the wetting and drying cycles are also defined in the volume $V$ and these cycles are imposed between extremely dry and wet conditions.

\footnotetext{
Corresponding author: kai.li@live.com
} 


\subsection{Mechanical analysis}

In this study, the authors assume that the studied elastoplastic structure meets the requirements of small displacements and small deformations.

In-situ stress analysis is firstly performed before shakedown modeling of the structure subjected to wetting and drying cycles. Considering the finite element calculation with the imposed boundary conditions, the stress state can be solved by linear elasticity:

$$
\sigma_{i j}^{*}(x, t)=D_{i j k l} \cdot\left[\varepsilon_{i j}^{*}(x, t)-\varepsilon_{i j}(x, 0)\right]
$$

in which $D_{i j k l}$ is the matrix of elastic moduli, $\sigma_{i j}^{*}(x, t)$ is the stress tensor and $\varepsilon_{i j}{ }^{*}(x, t)$ is the strain tensor. Here, the superscript $*$ represents the parameters calculated from mechanical analysis. With this calculation, the net mean stress for each point in the structure can be determined, which will be used in the following shakedown modeling.

\subsection{Real response of suction variation}

For the suction variation in the volume of structure, the real response of the studied structure can be written as follows:

$$
\varepsilon_{i j}(x, t)=M_{i j k l} \cdot s_{k l}(x, t)+\varepsilon_{i j}^{p}(x, t)+\varepsilon_{i j}^{I}(x, 0)
$$

where, $M_{i j k l}$ is the compliance elasticity matrix for suction loading, which regresses to $1 / E_{r}$ in terms of onedimensional case $\left(1 / E_{r}=A p+B\right)$. Additionally, $\varepsilon_{i j}^{p}(x, t)$ is the plastic strain tensor and the strain tensor $\varepsilon_{i j}(x, t)$ is kinematically admissible with $U_{j}(x, t)$ on $\Gamma_{U j}$.

In this equation, the suction tensor $s_{i j}(x, t)$ can be expressed by

$$
s_{i j}=\left(u_{a}-u_{w}\right) \cdot \delta_{i j}=s \cdot \delta_{i j}
$$

where, $\delta_{i j}$ is Kronecker delta whose value is 1 when $i=j$ and is equal to 0 otherwise.

\subsection{Elastic response of suction variation}

The response associated with the elastic part is expressed as follows:

$$
\varepsilon_{i j}^{e l}(x, t)=M_{i j k l} \cdot s_{k l}^{e l}(x, t)+\varepsilon_{i j}^{I}(x, 0)
$$

where, $I_{i j k l}$ is the identity tensor, $I_{i j k l}=1$ if $i=j=k=l$, and the strain tensor $\varepsilon_{i j}^{e l}(x, t)$ is kinematically admissible with $U_{j}(x, t)$ on $\Gamma_{U j}$.

Thus, the elasticity fields $U_{i}^{e l}(x, t)$ and $\varepsilon_{i j}^{e l}(x, t)$ can be calculated by an elastic analysis with the given boundary conditions and the compliance elasticity matrix for suction loading $M_{i j k l}$.

\subsection{Inelastic response of suction variation}

Because the general problem can be decomposed into elastic part and inelastic part, the inelastic strain can be expressed by the following equation:

$$
\varepsilon_{i j}^{i n e}(x, t)=\varepsilon_{i j}(x, t)-\varepsilon_{i j}^{e l}(x, t)
$$

where, $\varepsilon_{i j}^{i n e}(x, t)$ is kinematically admissible with 0 on $\Gamma_{U j}$.

Considering equations ( 2 and 4$)$, the equation 5 can be written as:

$$
\varepsilon_{i j}^{i n e}(x, t)=M_{i j k l} \cdot \rho_{k l}(x, t)+\varepsilon_{i j}^{p}(x, t)
$$

The residual field $\rho_{k l}(x, t)$ is obtained by the difference between total suction and elastic suction fields:

$$
\rho_{i j}(x, t)=s_{i j}(x, t)-s_{i j}^{e l}(x, t)
$$

where, $\rho_{l j}(x, t)$ is statically admissible with 0 in $V$.

As long as the plastic strain tensor $\varepsilon_{i j}^{p}(x, t)$ and the compliance elasticity matrix for suction loading $M_{i j k l}$ are known, the inelastic problem can be solved with null boundary condition and consequently the inelastic fields $U_{i}^{i n e}(x, t)$ and $\varepsilon_{i j}^{i n e}(x, t)$ are obtained.

Eventually, the residual field can be derived from equation 6 as follows:

$$
\rho_{i j}(x, t)=M_{i j k l}^{-1} \cdot\left[\varepsilon_{i j}^{i n e}(x, t)-\varepsilon_{i j}^{p}(x, t)\right]
$$

In the following section, the proposed method will be generalized to model the response of the full-scale structure.

\subsection{Structure with kinematic hardening}

The yield surface with a kinematic hardening can be defined by

$$
f=\sqrt{\left(s_{i j}-y_{i j}\right) \cdot\left(s_{i j}-y_{i j}\right)}-s_{\alpha}
$$

where, $s_{\alpha}$ is the threshold value of elastic limit for suction variation and $y_{i j}$ is a kinematic hardening tensor which can be related to the plastic strain,

$$
y_{i j}=h \cdot \varepsilon_{i j}^{p}
$$

where, $h$ is the kinematic hardening modulus, a critical important parameter in shakedown modeling. In this study, we consider $1 / h=C \cdot p+D$.

By rewriting equation 7 , the suction field can be expressed by:

$$
s_{i j}(x, t)=s_{i j}^{e l}(x, t)+\rho_{i j}(x, t)
$$

Here, the field of transformed structural parameters $Y_{i j}(\mathrm{x}, \mathrm{t})$ is defined:

$$
Y_{i j}(x, t)=y_{i j}(x, t)-\rho_{i j}(x, t)
$$

Taking into account the above equations, the yield surface can be expressed by:

$$
f\left(s_{i j}^{e l}-Y_{i j}\right) \leq 0
$$

This equation indicates that the yield surface is centered in $s_{i j}^{e l}$ and translates in the transformed structural parameter $Y_{i j}$ plane. 
With the transformed structural parameter field (equations 6, 10 and 12), the inelastic problem can be solved by

$$
\varepsilon_{i j}^{i n e}(x, t)=M_{i j k l}^{\prime} \cdot \rho_{k l}(x, t)+1 / h \cdot Y_{i j}(x, t)
$$

where, $M_{i j k l}$ with prime symbol is the modified compliance elasticity matrix for suction loading, defined by the following equation:

$$
M_{i j k l}^{\prime}=M_{i j k l}+1 / h \cdot I_{i j k l}
$$

where, $I_{i j k l}$ is the identity tensor, $I_{i j k l}=1$ if $i=j=k=l$.

Considering equation 14 , the residual field of the elasto-plastic structure is obtained:

$$
\rho_{i j}(x, t)=M_{i j k l}^{\prime-1} \cdot\left[\varepsilon_{i j}^{i n e}(x, t)-1 / h \cdot Y_{i j}(x, t)\right]
$$

Finally, the plastic strain field is given by combining equation 10 and 12 :

$$
\varepsilon_{i j}^{p}(x, t)=1 / h \cdot\left[Y_{i j}(x, t)+\rho_{i j}(x, t)\right]
$$

Consequently, a one-to-one relation exists between the kinematic hardening variable field $y_{i j}(x, t)$ and the transformed structural parameter field $Y_{i j}(x, t)$. At any time $t$, for a given kinematic hardening variable field $y_{i j}(x, t)$, there is a unique residual field $\rho_{i j}(x, t)$, a unique suction field $s_{i j}(x, t)$ as well as a unique transformed structural parameter $Y_{i j}(\mathrm{x}, \mathrm{t})$, and vice versa. Within this framework, the inelastic problem is solved through elastic analysis with a null boundary condition and a modified compliance elasticity matrix for suction loading. Eventually, all unknown fields at the limit state are obtained.

\subsection{Structure response under suction cycles}

During successive wetting and drying cycles, the elastic suction field can be expressed by:

$$
s_{i j}^{e l}(x, t)=[1-\wedge(t)] \cdot s_{i j_{\min }}^{e l}(x)+\wedge(t) \cdot s_{i j_{\max }}^{e l}(x)
$$

where, $\wedge(t)$ is a monotonic periodic function, varied between 0 and 1 .

The local suction at the level of the plastic mechanisms is expressed as:

$$
s_{i j}(x, t)=s_{i j}(x, t)-y_{i j}(x, t)
$$

In the local suction plane, the plasticity convex domains $C_{0}$ is a fixed segment on the isoclinic suction axis. The normality law is written with the Moreau's notation (Moreau 1971):

$$
\underset{l j}{\& g} \in \partial \phi_{C_{0}}\left(\xi_{i j}\right) \text { with } \xi_{i j} \in C_{0}
$$

where the plastic strain rate is an external normal to the convex C.

A loading collapse yield surface is presented in Figure 1, and it shows the increase of the preconsolidation stress $p_{i j}$ with the suction increase. In this study, no plastic deformation is generated by the mechanical loading during the wetting and drying cycles because the $p_{i j}$ value was selected less than $p_{i j}{ }^{*}$ representing the preconsolidation stress at the saturated state $($ suction $=0)$.

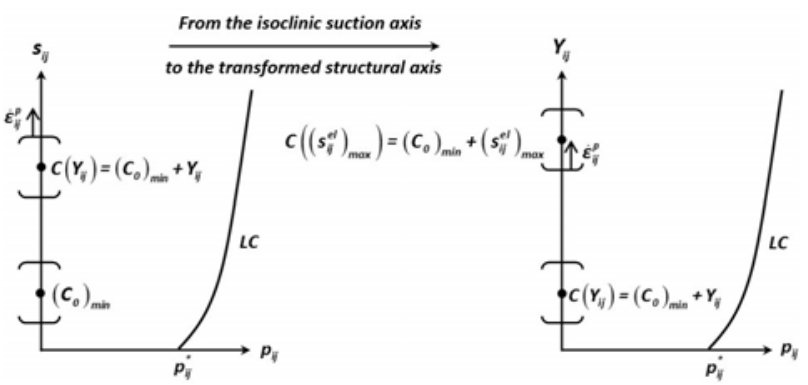

Fig. 1. Evolution of plasticity convex along the plane of $\left(s_{i j}-p_{i j}\right)$ and the plane of $\left(Y_{i j}-p_{i j}\right)$.

When the suction loading becomes very large, a stationary state can be reached and plastic shakedown is achieved. In this case, the distance between two extreme positions of the mobile convex in the transformed structural parameter plane can be obtained:

$$
\left\|\Delta Y_{i j}\right\|=\left\|s_{i j_{\max }^{e l}}\right\|-\left\|s_{i j_{\min }}^{e l}\right\|-2 \cdot s_{\alpha}
$$

Finally, the value of $\Delta Y_{i j}$ can be written as:

$$
\Delta Y_{i j}=\left(1-\frac{2 \cdot s_{\alpha}}{\left\|\Delta s_{i j}^{e l}\right\|}\right) \cdot \Delta s_{i j}^{e l}
$$

In these two equations, $\|\bullet\|$ is the notation of 2 norm.

\section{Numerical Simulation}

In this section, the displacement field of a natural expansive soil will be simulated after several wetting and drying cycles due to the variation of the climatic conditions, till the stabilized limit state. The finite element code CAST3M is exploited where the proposed shakedown-based model has been implemented.

- Studied material: artificially prepared mixure of $40 \%$ silt and $60 \%$ bentonite. The silt contains $60 \%$ quartz, $20 \%$ calcium montmorillonite, $11 \%$ feldspar and the remaining component was comprised of kaolinite and mica. The bentonite was comppsed of more than $90 \%$ of calcium montmorillonite. The initial dry density for the unsaturated expansive soil sample is $1.55 \mathrm{Mg} / \mathrm{m}^{3}$.

- Performed stress paths: a linear variatioin of suction with depth between 8 and $0 \mathrm{Mpa}$ is taken into account. Three constant foundation pressure on the boundary: 15, $30,60 \mathrm{kPa}$ are applied to the studied structure during suction cycles, respectively. The selected stresses are less than the preconsolidation stress at the saturated state, to prevent any plastic deformation due to mechanical loading.

- Numerical simulation: the geometry $(6 \mathrm{~m} \times 2 \mathrm{~m})$ is studied, made up of the aforementioned natural expansive soil. The lateral stress coefficient $K_{0}$ is used to show the applied lateral stress on the structure. Table 1 
presents the required parameters of the studied soils for the numerical simulation.

Table 1. Required parameters of the proposed model for studied sample.

\begin{tabular}{|c|c|c|c|}
\hline Parameter & Value & Parameter & Value \\
\hline$A\left(\mathrm{MPa}^{-2}\right)$ & $0.180 \mathrm{E}-1$ & $E(\mathrm{MPa})$ & 18 \\
\hline$B\left(\mathrm{MPa}^{-1}\right)$ & $0.182 \mathrm{E}-1$ & $v(-)$ & 0.2 \\
\hline$C\left(\mathrm{MPa}^{-2}\right)$ & $0.138 \mathrm{E}+0$ & $K_{0}(-)$ & 0.5 \\
\hline$D\left(\mathrm{MPa}^{-1}\right)$ & $-0.101 \mathrm{E}-1$ & & \\
\hline
\end{tabular}

- Simulation result:

Figure 2 describes the plastic strains on the soil surface after several wetting and drying cycles for the different vertical stresses. It can be observed that the vertical stress mainly influences the plastic strain field on the fondation area. Outside the fondation area, the swelling plastic strain reaches its maximum because of null external loads.

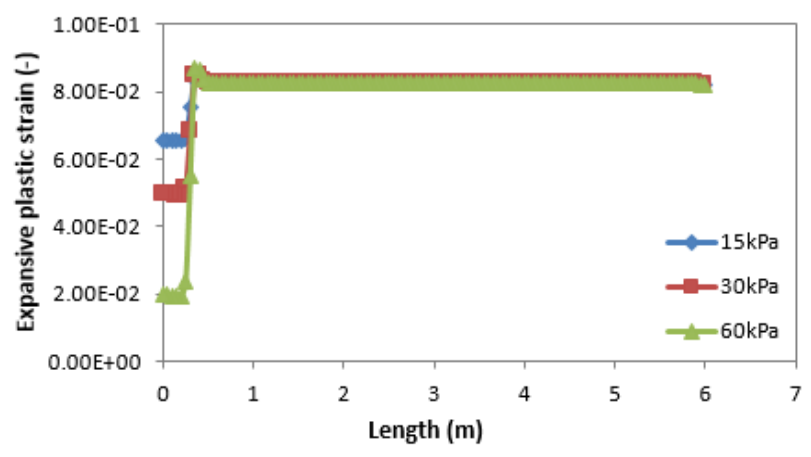

Fig. 2. Numerical simulation of plastic strain on the surface.

Figure 3 defines the inelastic displacement field of the geometry surface after wetting and drying cycles under the different foundation pressures. On the boundary of foundation, no settlement happens after the wetting and drying cycles and the heaving deformation decreases with the increase of the vertical overstress. All these findings can be related to the residual field in the studied geometry.

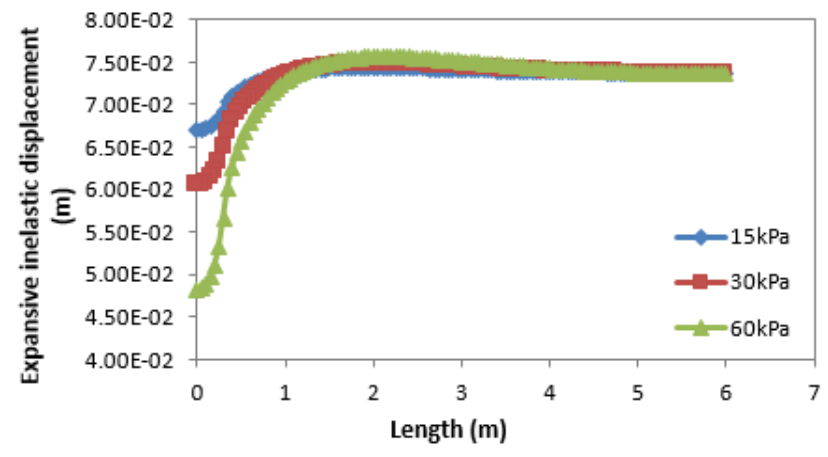

Fig. 3. Numerical simulation of inelastic displacement on the surface.

\section{Conclusion}

In this study, the finite element equations have been implemented in the calculation code CAST3M to perform the numerical simulation. In this simulation, an artificially expansive soil is used for shakedown analysis. From the modelling results, it can be observed that the swelling plastic strains and no settlement occure after drying and wetting cycles. All these findings can be explained by the existence of the residual stress field in the studied geometry.

\section{Acknowledgements}

This work is financially supported by the project of National Natural Science Foundation of China [Grant No: 51808306].

\section{References}

1. E.E. Alonso, J. Vaunat, A. Gens, Modeling the mechanical behavior of expansive clays. Engineering Geology, 54 (1999)

2. E.E. Alonso, E. Romero, C. Hoffmann, EG. Escudero, Expansive bentonite/sand mixture in cyclic controlled suction drying and wetting. Engineering Geology, 81(2005)

3. D. Masin, Double structure hydromechanical coupling formalism and a model for unsaturated expansive clays. Engineering Geology, 165 (2013)

4. K. Li., H. Nowamooz, C.Chazallon, B. Migault, Finite element modelling of the mechanical behaviour of unsaturated expansive soils subjected to wetting and drying cycles with shakedown concept, European journal of environmental and civil engineering, https://doi.org/10.1080/19648189.2017.1363666

5. V.G. Della, C. Jommi, E. Romero, A fully coupled elastic-plastic hydromechanical model for compacted soils accounting for clay activity. International Journal for Numerical and Analytical Methods in Geomechanics, 37, 5(2013)

6. E.E. Alonso, E. Romero, C. Hoffmann, Hydromechanical behavior of compacted granular expansive mixtures: experimental and constitutive study. Geotechnique, 61, 4(2011).

7. A. Gens, B. Vallejan, M. Sanchez, C. Imbert, MV. Villar, M. Van-Geet, Hydromechanical behavior of a heterogeneous compacted soil: experimental observations and modeling. Geotechnique, 61, 5(2011)

8. K. Li., H. Nowamooz, C.Chazallon, B. Migault, Mechanical behaviour of densely compacted expansive soils during wetting and drying cycles: an analytical model based on shakedown concept, European journal of environmental and civil engineering, https://doi.org/10.1080/19648189.2019.1568307 